\title{
Sorafenib in Japanese Patients with Locally Advanced or Metastatic Medullary Thyroid Carcinoma and Anaplastic Thyroid Carcinoma
}

\author{
Yasuhiro Ito, Naoyoshi Onoda, Ken-ichi Ito, Iwao Sugitani, ${ }^{4}$ Shunji Takahashi, \\ Iku Yamaguchi, Koki Kabu, and Katsuya Tsukada ${ }^{8}$
}

Background: Therapeutic options for treating advanced or metastatic medullary thyroid carcinoma (MTC) and anaplastic thyroid carcinoma (ATC) are still limited in Japan, even though vandetanib for MTC and lenvatinib for MTC and ATC have been approved. Sorafenib is an oral multikinase inhibitor approved for the treatment of patients with radioactive iodine-refractory differentiated thyroid cancer (DTC). An uncontrolled, open-label, multicenter, single-arm, Phase 2 clinical study was conducted to evaluate the safety and efficacy of sorafenib in Japanese patients with MTC and ATC.

Methods: Japanese patients with histologically confirmed ATC and locally advanced or metastatic MTC were enrolled from April to September 2014. The primary endpoint was to evaluate the safety of sorafenib. Treatment efficacy variables including progression-free survival (PFS), overall survival (OS), objective response rate (ORR), disease control rate (DCR), and maximum reduction in tumor size were evaluated as secondary endpoints. Patients received sorafenib $400 \mathrm{mg}$ orally twice daily on a continuous basis and then continued treatment until the occurrence of disease progression, unacceptable toxicity, or withdrawal of consent.

Results: A total of 20 patients were screened, and 18 ( 8 with MTC and 10 with ATC) were enrolled. The most common drug-related adverse events were palmar-plantar erythrodysesthesia (72\%), alopecia (56\%), hypertension (56\%), and diarrhea (44\%). In the ATC patients, median PFS was 2.8 months [confidence interval 0.7-5.6], and median OS was 5.0 months [confidence interval 0.7-5.7]; ORR and DCR were $0 \%$ and $40 \%$, respectively. In the MTC population, neither median PFS nor OS had been reached at the time of this analysis; ORR was 25\% and DCR was 75\%.

Conclusions: The toxicities reported in this study were consistent with the known safety profile of sorafenib. Sorafenib seems to be effective in the treatment of advanced MTC but not ATC, and could be a new treatment option for locally advanced or metastatic MTC and radioactive iodine-refractory DTC.

Keywords: anaplastic thyroid carcinoma, medullary thyroid carcinoma, overall survival, Phase II clinical trial, progression-free survival, sorafenib

\section{Introduction}

$\mathbf{T}$ HYROID CARCINOMA IS THE MOST frequent malignancy among malignant endocrine tumors, accounting for $3.8 \%$ of all malignant tumors. The number of new cases per 100,000 persons is 6.9 and 20.6 for men and women, respectively (1).
Based on histologic subtype, thyroid carcinoma can be classified as either differentiated carcinoma (DTC), including papillary and follicular carcinomas; medullary thyroid carcinoma (MTC); or anaplastic thyroid carcinoma (ATC). DTC and ATC are derived from thyroid follicular epithelial cells, whereas MTC is derived from the parafollicular cells (C

\footnotetext{
${ }^{1}$ Clinical Trial Management Center, Kuma Hospital, Kobe, Japan.

${ }^{2}$ Department of Surgical Oncology, Osaka City University Graduate School of Medicine, Osaka, Japan.

${ }^{3}$ Department of Surgery, Shinshu University School of Medicine, Matsumoto, Japan.

${ }^{4}$ Department of Endocrine Surgery II, Nippon Medical School, Tokyo, Japan.

${ }^{5}$ Department of Medical Oncology, Cancer Institute Hospital, Japanese Foundation for Cancer Research, Tokyo, Japan.

${ }^{6}$ Product Development, Clinical Statistics, Bayer Yakuhin Ltd., Osaka, Japan.

${ }^{7}$ Medical Affairs/KI Oncology; ${ }^{8}$ Medical Affairs, Oncology and Hematology, and Clinical Development, Specialty Medicine; Bayer Yakuhin Ltd., Tokyo, Japan.

(C) Yasuhiro Ito et al. 2017; Published by Mary Ann Liebert, Inc. This is an Open Access article distributed under the terms of the Creative Commons Attribution License, which permits unrestricted use, distribution, and reproduction in any medium, provided the original work is properly cited.
} 
cells) that secrete calcitonin (2). In Japan, the 2004 incidence rates of thyroid carcinoma classified by histologic subtypes were $92.5 \%$ papillary thyroid carcinoma, $4.8 \%$ follicular thyroid carcinoma, $1.4 \%$ ATC, $1.3 \%$ MTC, and $0 \%$ other (3).

For recurrent or advanced MTC, systemic chemotherapy, including doxorubicin, cis-platinum, 5-fluorouracil, vincristine, dacarbazine, and streptozocin, has been tested, but the efficacy was limited (4). Because patients with ATC are always assessed as stage IV in the TNM/Union for International Cancer Control system at the time of diagnosis and have an extremely poor prognosis (5), multimodality therapy comprising surgery, chemotherapy, and external beam irradiation therapy is necessary. ATC is generally resistant to treatment because of its high-grade malignancy (6-8), although weekly paclitaxel was reported to be somewhat effective (9).

Recent clinical trials have assessed the use of tyrosine kinase inhibitors (TKIs) for the treatment of MTC and ATC. Vandetanib has been adopted as a treatment option for MTC (10), and lenvatinib was approved in Japan for the treatment of MTC and ATC based on the results of a Phase 2 study in a small number of Japanese patients (11). Cabozantinib, which has been approved by the U.S. Food and Drug Administration (FDA) and the European Medicines Agency (EMA) for treating advanced MTC, is not approved in Japan.

Sorafenib inhibits vascular endothelial growth factor receptors (VEGFR) 1, 2, and 3; platelet-derived growth factor receptor; mitogen-activated protein kinase (MAPK) members BRAF and RET; chimeric protein RET/PTC; and c-Kit. Sorafenib was the first TKI showing a prolongation of progressionfree survival (PFS) of radioactive iodine-refractory DTC patients based on an international, collaborative, randomized, placebo-controlled Phase 3 study (DECISION; $N=419$ ) (12). Sorafenib has been shown to inhibit the proliferation and angiogenesis of DTC and MTC in an in vivo mouse model with carcinoma transplants (13-16). In the current Phase 2 study, the safety and efficacy of sorafenib were evaluated in Japanese patients with MTC and ATC.

\section{Materials and Methods}

\section{Study design and patients}

This uncontrolled, open-label, multicenter, single-arm, Phase 2 clinical study evaluated the safety and efficacy of sorafenib in Japanese patients with ATC and MTC. Japanese patients who met all of the following key inclusion criteria were enrolled: age $\geq 18$ years; histologically or cytologically confirmed ATC/locally advanced or metastatic MTC; a measurable lesion as assessed by Response Evaluation Criteria in Solid Tumors (RECIST) v1.1 or a clinically evaluable unmeasurable lesion; Eastern Cooperative Oncology Group Performance Status (ECOG PS) score of 0 or 1; adequate bone marrow, liver, and renal function; and an expected survival of $\geq 12$ weeks. Patients who met any of the following exclusion criteria were not enrolled: previous or concurrent malignancies other than thyroid cancer within five years prior to enrollment in this study; previous anticancer treatment with TKIs, monoclonal antibody-targeted VEGF or VEGFR (including approved and investigational drugs), other molecular targeted therapies, or more than one chemotherapy regimen; complication with or history of hemorrhagic diathesis or coagulation disorder; infiltration of tumor into the trachea, bronchus, or esophagus with a risk for severe bleeding not treated locally before enrollment; congestive heart failure of New York Heart Association class $\geq$ III or unstable angina; uncontrolled hypertension (systolic blood pressure $>150 \mathrm{mmHg}$ or diastolic blood pressure $>90 \mathrm{mmHg}$ ) despite appropriate drug therapy; kidney dialysis; and intake of strong CYP3A4 inducers (including St. John's wort, dexamethasone $\geq 16 \mathrm{mg} /$ day, phenytoin, carbamazepine, rifampicin, rifabutin, and phenobarbital) within 7 days of the initiation of treatment with sorafenib. All patients signed informed consent before initiating any procedures.

\section{Procedure}

Patients received sorafenib orally at $800 \mathrm{mg}$ (two $400 \mathrm{mg}$ doses) daily at $12 \mathrm{~h}$ intervals on a continuous basis. One cycle consisted of 28 days. Dose reduction (to 600, 400, and $200 \mathrm{mg}$ ) and dose interruption were allowed based on adverse event (AE) grade when AEs were determined by the investigator to be related to sorafenib. The grade of the AE was determined according to the National Cancer Institute Common Terminology Criteria for Adverse Events v4.03. Patients continued treatment until disease progression, unacceptable toxicity, or withdrawal of consent. Continued treatment with sorafenib was allowed, even after disease progression, if it was considered beneficial by the investigators. Objective responses were evaluated using RECIST. Images of the neck, chest, abdomen, pelvis, and brain were obtained using computed tomography (CT) or magnetic resonance imaging (MRI) 28 days before the initiation of treatment, every two cycles after the initiation of treatment, and within 30 days after study discontinuation.

\section{Outcomes}

The primary endpoint was to assess the safety of sorafenib, which was monitored until the end of cycle 6 and every second cycle thereafter. The treatment efficacy variables PFS, overall survival (OS), objective response rate, disease control rate (DCR), and maximum reduction in tumor size were evaluated as secondary endpoints. CT scan or MRI were evaluated every second cycle (every 56 days). OS was captured after patients discontinued treatment because of disease progression, and their survival status was evaluated every three months.

The imaging analysis was used to assess tumor response in accordance with RECIST.

\section{Statistical analysis}

Because patients with MTC and ATC in Japan account for $<2 \%$ of those with thyroid carcinoma, the sample size was not based on statistical calculation but based on the feasibility of collecting patients. Accordingly, the sample size was determined to be 16 patients. Efficacy was evaluated in the full analysis set, which included patients who received sorafenib at least once and had sufficient data for efficacy analysis. Kaplan-Meier estimates and curves were obtained for PFS and OS in patients with MTC and ATC. This study was registered at Clinical Trials.gov (NCT02114658).

\section{Results \\ Patients}

A total of 20 patients (10 patients each with MTC and ATC) were screened at five study sites in Japan from April to 
September 2014. Of 20 patients screened, two patients who did not meet the inclusion criteria were not treated and were excluded from the safety and efficacy analyses (one patient had no measurable or an unmeasurable lesion, and the other patient had a total bilirubin $\geq 1.5 \times$ the upper limit of normal). Therefore, safety and efficacy were evaluated in eight patients with MTC and 10 patients with ATC (Table 1). Baseline patient demographic characteristics are described in Table 1. The most common locations of metastases were the lungs ( 6 patients; $33 \%$ ), mediastinal lymph node (5 patients; $28 \%$ ), and thyroid gland (5 patients; $28 \%$ ). Four patients ( 2 with ATC and 2 with MTC) had only clinically evaluable unmeasurable lesions. These lesions were located in the lungs with multiple small miliary metastases. Tumor progression in these four patients was clinically evident by the appearance of clinical symptoms such as dyspnea.

\section{Treatment and dose modifications}

The mean dose, median treatment duration (including time of dose interruption), number of patients affected by dose reduction, reasons for dose reduction, and permanent discontinuation are summarized in Table 2. Overall, the mean daily sorafenib dose in all patients was $530 \pm 167 \mathrm{mg} /$ day, and median treatment duration in all patients was 15.5 weeks (range 2.9-40.0 weeks). Of the 18 patients, 14 (78\%) had one or more sorafenib dose reduction. Primary reasons for dose reduction were palmar-plantar erythrodysesthesia syndrome (8 patients; 44\%), hypophosphatemia (2 patients; 11\%), proteinuria (2 patients; $11 \%$ ), and maculopapular rash (2 patients; 11\%). Drug interruptions were observed in $12(67 \%)$ patients and were primarily caused by palmar-plantar erythrodysesthesia (7 patients [39\%]; 5 [63\%] with MTC and 2 [20\%] with ATC).

\section{Safety}

The most common treatment-emergent $\mathrm{AE}$ of any grade was palmar-plantar erythrodysesthesia, which was observed in $13(72 \%)$ patients; other treatment-emergent AEs included alopecia (10 patients; $56 \%$ ), hypertension (10 patients; $56 \%$ ), and diarrhea (8 patients; 44\%; Table 3). The most frequent AEs of grade $\geq 3$ were palmar-plantar erythrodysesthesia (22\%) and an aspartate aminotransferase (AST) increase $(22 \%)$. Grade 4 alanine aminotransferase (ALT) increases were observed in three (17\%) patients, and three grade 5 AEs were observed in three patients $(17 \% ; 3$ [30\%] with ATC). These included disseminated intravascular coagulation, tumor bleeding, and tracheal stenosis in one patient each, which were due to progression of underlying disease and unrelated to sorafenib (data not shown).

\section{Efficacy}

In patients with MTC, complete response (CR), partial response (PR), and stable disease (SD) were observed in 0,2 $(25 \%)$, and $4(50 \%)$ patients, respectively (Table 4$)$. The response rate (ORR; CR + PR) was $25 \%$ and the DCR (CR + $\mathrm{PR}+\mathrm{SD}$ ) was $75 \%$ (Table 4). CR, PR, and SD were observed

Table 1. Patient Background

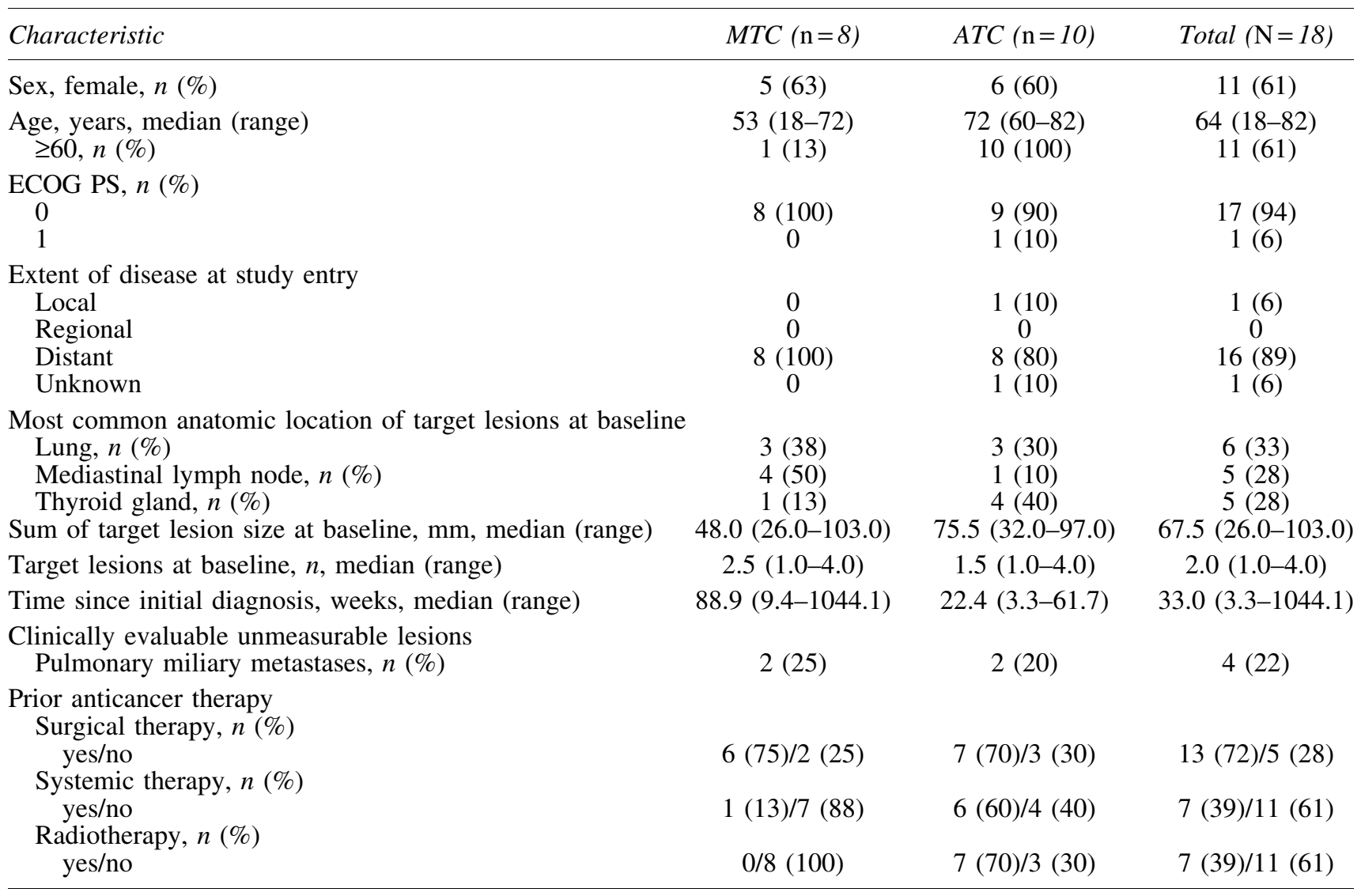

ATC, anaplastic thyroid carcinoma; ECOG PS, Eastern Cooperative Oncology Group Performance Status; MTC, medullary thyroid carcinoma. 
Table 2. Treatment and Dose Modifications

\begin{tabular}{|c|c|c|c|}
\hline & $\operatorname{MTC}(\mathrm{n}=8)$ & $\operatorname{ATC}(\mathrm{n}=10)$ & Total $(\mathrm{N}=18)$ \\
\hline Mean dose $\pm S D$, mg & $456 \pm 191$ & $590 \pm 124$ & $530 \pm 167$ \\
\hline $\begin{array}{l}\text { Treatment duration, including time interrupted, } \\
\text { weeks, median (range) }\end{array}$ & $24.0(12.3-40.0)$ & $10.5(2.9-32.1)$ & $15.5(2.9-40.0)$ \\
\hline Dose reduction, $n(\%)$ & $7(88)$ & $7(70)$ & $14(78)$ \\
\hline \multicolumn{4}{|l|}{ Reason for dose reduction, $n(\%)$} \\
\hline Palmar-plantar erythrodysesthesia & $5(63)$ & $3(30)$ & $8(44)$ \\
\hline Hypophosphatemia & $1(13)$ & $1(10)$ & $2(11)$ \\
\hline Proteinuria & $1(13)$ & $1(10)$ & $2(11)$ \\
\hline Rash maculopapular & $2(25)$ & 0 & $2(11)$ \\
\hline Alopecia & $1(13)$ & 0 & $1(6)$ \\
\hline Diarrhea & $1(13)$ & 0 & $1(6)$ \\
\hline Decreased appetite & 0 & $1(10)$ & $1(6)$ \\
\hline Dysphagia & 0 & $1(10)$ & $1(6)$ \\
\hline Fatigue & 0 & $1(10)$ & $1(6)$ \\
\hline Hypertension & 0 & $1(10)$ & $1(6)$ \\
\hline Nausea & 0 & $1(10)$ & $1(6)$ \\
\hline Vomiting & 0 & $1(10)$ & $1(6)$ \\
\hline Drug interruption, $n(\%)$ & $6(75)$ & $6(60)$ & $12(67)$ \\
\hline \multicolumn{4}{|l|}{ Reason for drug interruption } \\
\hline Palmar-plantar erythrodysesthesia syndrome & $5(63)$ & $2(20)$ & 7 (39) \\
\hline ALT increased & $2(25)$ & 0 & $2(11)$ \\
\hline AST increased & $1(13)$ & $1(10)$ & $2(11)$ \\
\hline Hypertension & 0 & $2(20)$ & $2(11)$ \\
\hline Rash maculopapular & $2(25)$ & 0 & $2(11)$ \\
\hline Arthritis & 0 & $1(10)$ & $1(6)$ \\
\hline Decreased appetite & 0 & $1(10)$ & $1(6)$ \\
\hline Dysphagia & 0 & $1(10)$ & $1(6)$ \\
\hline Hypophosphatemia & 0 & $1(10)$ & $1(6)$ \\
\hline Esophageal stenosis & 0 & $1(10)$ & $1(6)$ \\
\hline Tumor hemorrhage & 0 & $1(10)$ & $1(6)$ \\
\hline Permanent discontinuation, $n(\%)$ & $2(25)$ & $1(10)$ & $3(17)$ \\
\hline \multicolumn{4}{|l|}{ Reason for permanent discontinuation, $n(\%)$} \\
\hline ALT increased & $2(25)$ & $1(10)$ & $3(17)$ \\
\hline AST increased & $1(13)$ & 0 & $1(6)$ \\
\hline
\end{tabular}

ALT, alanine aminotransferase; AST, aspartate aminotransferase.

in 0,0 , and $4(40 \%)$ of ATC patients, respectively; ORR was 0 and the DCR was $40 \%$. The best overall response in four patients without a target lesion (2 patients each with MTC and ATC) was non-CR/non-progressive disease (PD;
Table 4). At the time of data cutoff, PD or death was observed in 10 patients $(56 \% ; 1$ [13\%] with MTC and 9 [90\%] with ATC; data not shown). The median PFS in patients with MTC had not yet been reached [confidence interval (CI) 5.6

Table 3. Adverse Events of Any Grade Occurring in $\geq 20 \%$ of Patients During the Treatment Period

\begin{tabular}{|c|c|c|c|c|c|c|}
\hline \multirow[b]{2}{*}{ Adverse events, n (\%) } & \multicolumn{2}{|c|}{$\operatorname{MTC}(\mathrm{n}=8)$} & \multicolumn{2}{|c|}{$A T C(\mathrm{n}=10)$} & \multicolumn{2}{|c|}{ Total $(\mathrm{N}=18)$} \\
\hline & Any grade & Grade $\geq 3$ & Any grade & Grade $\geq 3$ & Any grade & Grade $\geq 3$ \\
\hline Palmar-plantar erythrodysesthesia & $8(100)$ & $4(50)$ & $5(50)$ & 0 & $13(72)$ & $4(22)$ \\
\hline Alopecia & $7(88)$ & 0 & $3(30)$ & 0 & $10(56)$ & 0 \\
\hline Hypertension & $5(63)$ & $1(13)$ & $5(50)$ & $2(20)$ & $10(56)$ & $3(17)$ \\
\hline Diarrhea & $5(63)$ & 0 & $3(30)$ & 0 & 8 (44) & 0 \\
\hline Stomatitis & $5(63)$ & 0 & $2(20)$ & 0 & $7(39)$ & 0 \\
\hline Rash & $2(25)$ & 0 & $4(40)$ & 0 & $6(33)$ & 0 \\
\hline Rash maculopapular & $3(38)$ & $1(13)$ & $3(30)$ & 0 & $6(33)$ & $1(6)$ \\
\hline Blood TSH increased & $3(38)$ & 0 & $3(30)$ & 0 & $6(33)$ & 0 \\
\hline ALT increased & $3(38)$ & $2(25)$ & $2(20)$ & $1(10)$ & $5(28)$ & 3 (17) \\
\hline Weight decreased & 0 & 0 & $5(50)$ & 0 & $5(28)$ & 0 \\
\hline Headache & $3(38)$ & 0 & $2(20)$ & 0 & $5(28)$ & 0 \\
\hline AST increased & $2(25)$ & $2(25)$ & $2(20)$ & $2(20)$ & $4(22)$ & $4(22)$ \\
\hline Fatigue & $1(13)$ & 0 & $3(30)$ & 0 & $4(22)$ & 0 \\
\hline
\end{tabular}

TSH, thyrotropin. 
Table 4. Summary of Efficacy Results

\begin{tabular}{lcc}
\hline & $M T C(\mathrm{n}=8)$ & $A T C(\mathrm{n}=10)$ \\
\hline Median PFS, months [CI] & NE [5.6-NE] & $2.8[0.7-5.6]$ \\
Median OS, months [CI] & NE [NE-NE] & $5.0[0.7-5.7]$ \\
Best overall response & & \\
CR, $n(\%)$ & 0 & 0 \\
PR, $n(\%)$ & $2(25)$ & 0 \\
SD, $n(\%)$ & $4(50)$ & $4(40)$ \\
Non-CR/non-PD, ${ }^{\mathrm{a}} n(\%)$ & $2(25)$ & $2(20)$ \\
PD, $n(\%)$ & 0 & 0 \\
Not evaluable ${ }^{\mathrm{b}}{ }^{n} n(\%)$ & 0 & $4(40)$ \\
ORR, ${ }^{\mathrm{c}} n(\%)$ & $2(25)$ & 0 \\
DCR $^{\mathrm{d}} n(\%)$ & $6(75)$ & $4(40)$ \\
\hline
\end{tabular}

${ }^{\mathrm{a}}$ Patients who had no target lesion, without unequivocal CR or PD. ${ }^{\mathrm{b}}$ Patients died before tumor assessment or did not meet SD criteria. ${ }^{\mathrm{c}} \mathrm{ORR}=\mathrm{CR}+\mathrm{PR}$

${ }^{\mathrm{d}} \mathrm{DCR}=\mathrm{CR}+\mathrm{PR}+\mathrm{SD}$.

$\mathrm{CR}$, complete response; DCR, disease control rate; NE, not estimable; ORR, partial response; OS, overall survival; PD, progressive disease; PFS, progression-free survival; PR, partial response; SD, stable disease.

months-NE]; Fig. 1A), and in patients with ATC, it was 2.8 months ([CI 0.7-5.6]; Fig. 1B). The median [CI] OS in patients with MTC had not yet been reached ([CI NE-NE]; Fig. 1C), and in patients with ATC, it was 5.0 months ([CI 0.7-5.7]; Table 4). The rate of PFS at six months in patients with MTC was 86\% [CI 33-98\%], and in patients with ATC, it was $11 \%$ [CI $1-39 \%$ ]. The rate of OS at six months in patients with MTC was 100\% [CI 100-100\%], and in the patients with ATC, it was 20\% ([CI 3-47\%; Fig. 1D).

The maximum reduction in size of the target lesion from baseline in each patient is shown in Figure 1E. Seven patients were excluded from the imaging assessment, including four who had no target lesions (2 each for MTC and ATC) and three patients with ATC who died before the first scheduled assessment after sorafenib administration. Overall, 39\% of patients (7/18; 75\% [6/8] with MTC and 10\% [1/10] with ATC) showed reductions in target lesion size. Two patients with MTC had a reduction $\geq 30 \%$, and no patients had an increase. None of the patients with ATC had a reduction $\geq 10 \%$, one patient had a reduction between $0 \%$ and $10 \%$, and four patients had increases in target lesion size.

\section{Discussion}

When the present study was initiated, the only available treatments for patients with MTC and ATC were surgical resection and radiation therapy. Systemic therapies, including chemotherapy and treatment with somatostatin derivatives, were available for patients with recurrent MTC (6), and doxorubicin and cisplatin were available for patients with recurrent ATC (17). However, the efficacy of these treatments was shown to be limited. After this study was initiated, lenvatinib and vandetanib were approved for the treatment of thyroid carcinomas (DTC, MTC, and ATC), but cabozantinib, which has been approved by the FDA in the United States and by the EMA in Europe for treating advanced MTC, was not approved in Japan. In order to increase the number of treatment options for MTC and ATC patients, it is also critical to evaluate the efficacy and safety of sorafenib. Assess- ment of safety was the primary endpoint in this Phase II study, as requested by the Japan health authority.

Evaluation of the primary outcome, safety, showed that toxicities were observed in all patients. The most frequent AEs included palmar-plantar erythrodysesthesia in $72 \%$ of patients, alopecia in $56 \%$, hypertension in $56 \%$, and diarrhea in $44 \%$. The most frequent grade 3 or 4 AEs included palmarplantar erythrodysesthesia in $22 \%$ of patients, AST increase in $22 \%$, hypertension in $17 \%$, and ALT increase in $17 \%$. A dose reduction was observed in 14 patients (7 MTC and 7 ATC) due to AEs, and 12 patients (6 MTC and 6 ATC) interrupted the treatment due to AEs. Although permanent treatment discontinuation was observed in three patients (2 MTC and 1 ATC) due to grade 3 or 4 hepatic toxicity, all three patients immediately recovered after discontinuation of the drug. Overall, treatment with sorafenib was well tolerated. In the DECISION study, which evaluated the efficacy of sorafenib in patients with advanced or metastatic DTC, it was reported that all grades of treatment-emergent AEs were observed as follows: palmar-plantar erythrodysesthesia in $76.3 \%$ of patients, alopecia in $67.1 \%$, hypertension in $40.6 \%$, and diarrhea in $68.6 \%$ (12). In a previous Phase 2 study of sorafenib for the treatment of MTC or ATC, common AEs in MTC patients included hand-foot syndrome (HFS; 75-90\%), weight loss $(48-67 \%)$, fatigue $(2533 \%)$, and diarrhea (16$81 \%)(18,19)$; common AEs in ATC patients included HFS (79\%), weight loss (29-60\%), fatigue (59-60\%), and diarrhea $(35-77 \%)(20,21)$. The present safety results are consistent with those of the DECISION study, although the relative frequency of the various AEs was different to some extent because of the small sample size in the current study. In the present study, grade 5 AEs were observed in three ATC patients (disseminated intravascular coagulation, tumor bleeding, and tracheal stenosis in one patient each), but they were consequences of worsening of the underlying disease and were not considered to be drug-related by the investigators. In particular, tumor bleeding, which infiltrated into the right common carotid artery before sorafenib initiation, was considered to be a result of worsening of primary disease. Serious drug-related AEs of ALT and AST increase and anorexia were reported in three patients ( 2 with MTC and 1 with ATC), but these events were resolved. All AEs observed in the present study were manageable by dose adjustments and symptomatic treatments. No new safety concerns related to vital signs, electrocardiogram results, or ECOG PS scores were found. The mean daily sorafenib dose in patients with ATC $(590 \mathrm{mg} /$ day) was higher than that in patients with MTC ( $456 \mathrm{mg} / \mathrm{day}$ ) because the dose adjustments were less frequent in the ATC population. ATC patients showed faster progression and shorter time to discontinuation compared to MTC patients.

In a previous Phase 2 study in patients with metastatic and locally advanced sporadic MTC, Lam et al. reported that PR and SD were observed in $6 \%(n=1)$ and $88 \%(n=14)$, respectively, of the 16 patients (19). Ahmed et al. reported a Phase 2 study in 15 patients with metastatic and locally advanced MTC in which PR in two (13\%) patients and SD in 13 $(87 \%)$ patients were observed six months after the initiation of treatment with sorafenib (20). The response rates in patients with MTC in the present study are similar to those in previous studies. Although Savvides et al. have reported that PR was observed in 2/20 (10.0\%) patients and SD was 
A

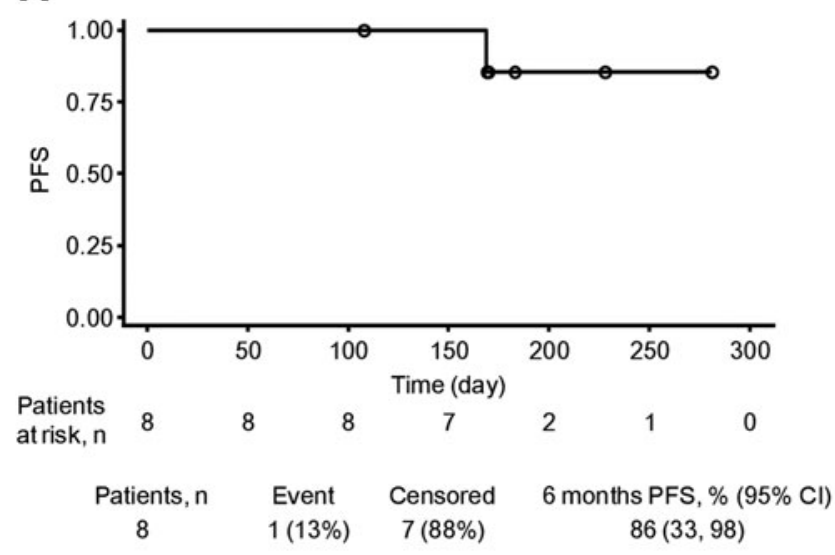

C

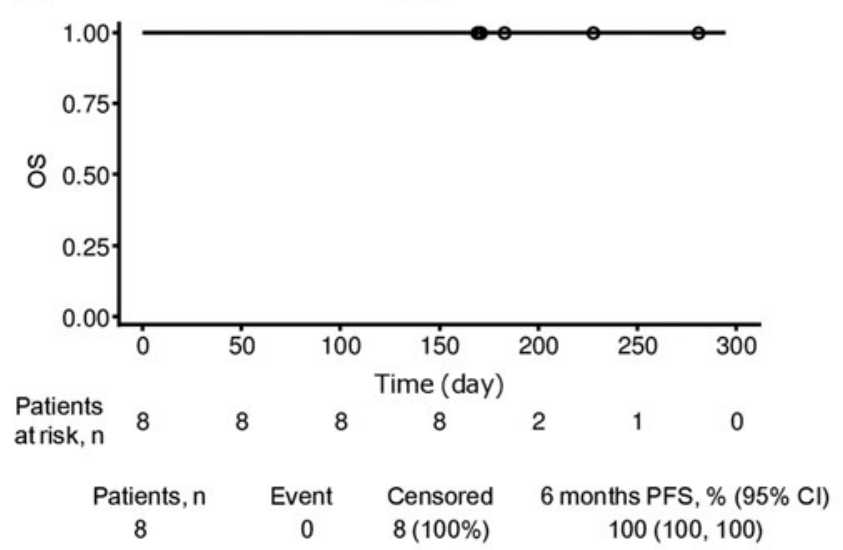

B

B ATC

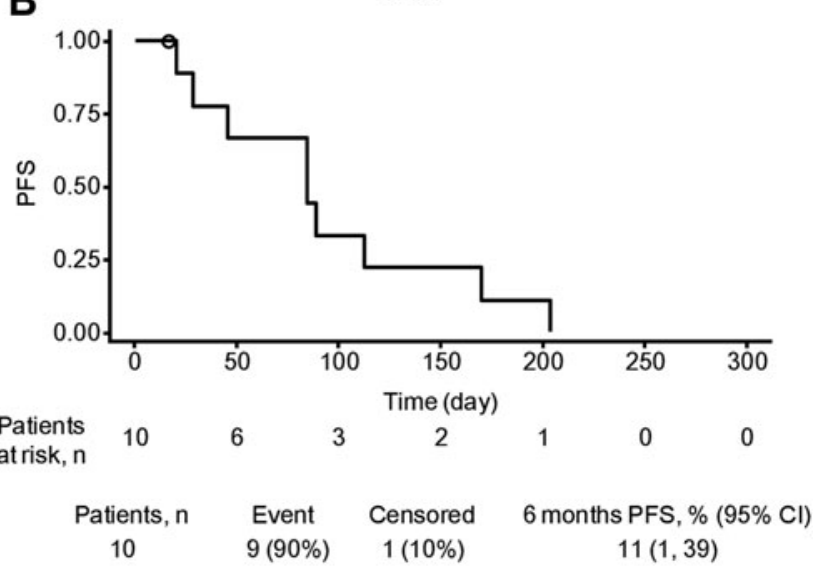

D ATC

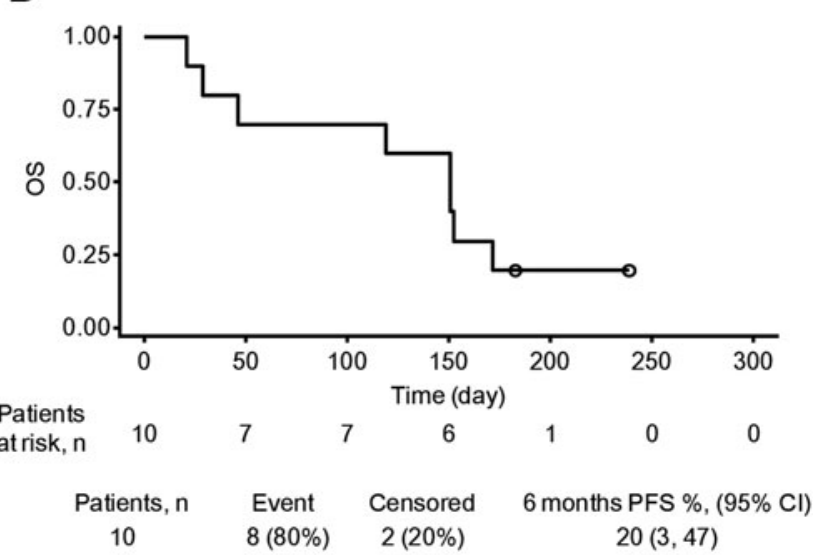

E

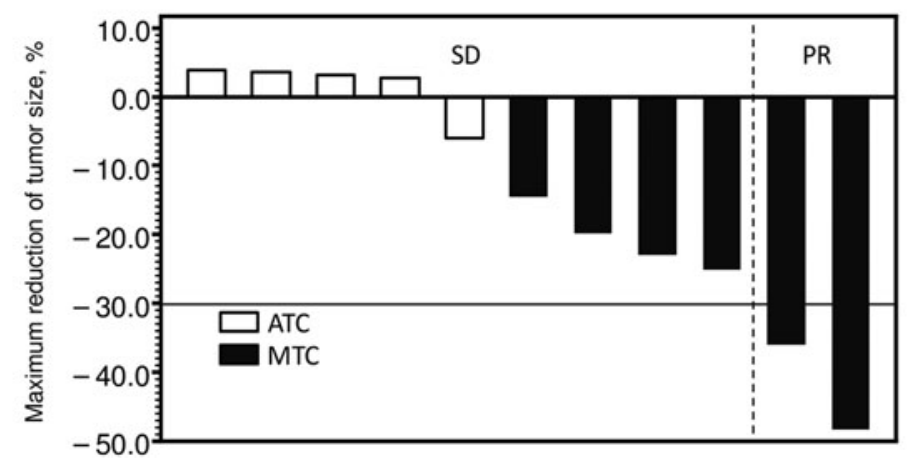

FIG. 1. Kaplan-Meier curve showing PFS for MTC (A) and ATC (B), and OS for MTC (C) and ATC (D). Waterfall plot of best tumor response showing maximum shrinkage of target lesions (E). Closed and open bars stand for MTC and ATC, respectively. ATC, anaplastic thyroid carcinoma; MTC, medullary thyroid carcinoma; OS, overall survival; PFS, progression-free survival; $\mathrm{PR}$, partial response; SD, stable disease.

observed in 5/18 (28.0\%) patients in a Phase 2 study in ATC patients (21), PR was not observed in patients with ATC in the present study. However, in the present study, median OS in patients with ATC was 151 days (5.0 months), which was longer than that reported for 547 patients with common type ATC (113 days, 3.7 months) in a survey evaluating the prognosis of 677 Japanese patients with ATC conducted by the ATC Research Consortium of Japan (22).

The results of this study should be interpreted in consideration of the potential detection bias and/or risk of perfor- mance bias. To elucidate the effects of sorafenib on MTC and ATC more accurately, a large number of patients, based on a statistical sample calculation, would be required in the future. In addition, an appropriate interval for the radiologic evaluation should be considered especially for ATC patients, because three ATC patients in this study died before the first scheduled assessment and the tumor response was not evaluated.

In conclusion, treatment with sorafenib was well tolerated in patients with advanced and metastatic MTC or ATC, and the safety profile was similar to that reported for other 
indications such as renal cell and hepatic cell carcinomas. Overall, sorafenib appears to be a possible new treatment option in patients with MTC.

\section{Acknowledgments}

The authors appreciate the cooperation and contribution of everyone involved in this study, including the study centers and all of the patients and their families. This study was funded by Bayer Yakuhin Ltd.

\section{Author Disclosure Statement}

K.K., I.Y., and K.T. are employees of Bayer Yakuhin Ltd. No competing financial interests exist for the remaining authors.

\section{References}

1. National Cancer Institute. Surveillance, Epidemiology, and End Results Program. Available at: https://seer.cancer.gov/ statfacts/html/thyro.html (accessed February 20, 2017).

2. American Cancer Society. What is thyroid cancer? Available at: www.cancer.org/cancer/thyroidcancer/detailedguide/ thyroid-cancer-what-is-thyroid-cancer (accessed November 10, 2016).

3. Saikawa M, Haruki A 2013 Column 2. Prevalence of various histological types of thyroid cancer. In: Takami $\mathrm{H}$, Ito Y, Noguchi H, Yoshida A, Okamoto T (eds) Treatment of Thyroid Tumor: Japanese Clinical Guidelines. Spinger Japan, p. 27.

4. Orlandi F, Caraci P, Mussa A, Saggiorato E, Pancani G, Angeli A 2001 Treatment of medullary thyroid carcinoma: an update. Endocr Relat Cancer 8:135-147.

5. Union for International Cancer Control 2009 TNM Classification of Malignant Tumors. Seventh edition. WileyBlackwell, Hoboken, NJ.

6. Haymart MR, Banerjee M, Yin H, Worden F, Griggs JJ 2013 Marginal treatment benefit in anaplastic thyroid cancer. Cancer 119:3133-3139.

7. Smallridge RC 2012 Approach to the patient with anaplastic thyroid carcinoma. J Clin Endocrinol Metab 97:2566-2572.

8. Sugino K, Onoda N 2013 Part VII: anaplastic carcinoma. In: Takami H, Ito Y, Noguchi H, Yoshida A, Okamoto T (eds) Treatment of Thyroid Tumor: Japanese Clinical Guidelines. Vol. 1. Springer, Tokyo, Japan.

9. Higashiyama T, Ito Y, Hirokawa M, Fukushima M, Uruno T, Miya A, Matsuzuka F, Miyauchi A 2010 Induction chemotherapy with weekly paclitaxel administration for anaplastic thyroid carcinoma. Thyroid 20:7-14.

10. Wells SA Jr, Robinson BG, Gagel RF, Dralle H, Fagin JA, Santoro M, Baudin E, Elisei R, Jarzab B, Vasselli JR, Read J, Langmuir P, Ryan AJ, Schlumberger MJ 2012 Vandetanib in patients with locally advanced or metastatic medullary thyroid cancer: a randomized, double-blind Phase III trial. J Clin Oncol 30:134-141.

11. Takahashi S, Kiyota N, Yamazaki T, Chayahara N, Nakano K, Inagaki L, Toda K, Enokida T, Minami H, Imamura Y, Sasaki T, Suzuki T, Fujino K, Dutcus C, Tahara M 2016 Phase 2 study of lenvatinib in patients with differentiated, medullary, and anaplastic thyroid cancer: final analysis results [abstract]. J Clin Oncol 34:6088.

12. Brose MS, Nutting CM, Jarzab B, Elisei R, Siena S, Bastholt L, de la Fouchardiere C, Pacini F, Paschke R, Shong YK, Sherman SI, Smit JW, Chung J, Kappeler C, Pena C, Molnar I, Schlumberger MJ, Decision Investigators 2014
Sorafenib in radioactive iodine-refractory, locally advanced or metastatic differentiated thyroid cancer: a randomised, double-blind, Phase 3 trial. Lancet 384:319-328.

13. Carlomagno F, Anaganti S, Guida T, Salvatore G, Troncone G, Wilhelm SM, Santoro M 2006 BAY 43-9006 inhibition of oncogenic RET mutants. J Natl Cancer Inst 98:326-334.

14. Henderson YC, Ahn SH, Kang Y, Clayman GL 2008 Sorafenib potently inhibits papillary thyroid carcinomas harboring RET/PTC1 rearrangement. Clin Cancer Res 14:4908-4914.

15. Plaza-Menacho I, Mologni L, Sala E, Gambacorti-Passerini C, Magee AI, Links TP, Hofstra RM, Barford D, Isacke CM 2007 Sorafenib functions to potently suppress RET tyrosine kinase activity by direct enzymatic inhibition and promoting RET lysosomal degradation independent of proteasomal targeting. J Biol Chem 282:29230-29240.

16. Wilhelm SM, Carter C, Tang L, Wilkie D, McNabola A, Rong H, Chen C, Zhang X, Vincent P, McHugh M, Cao Y, Shujath J, Gawlak S, Eveleigh D, Rowley B, Liu L, Adnane L, Lynch M, Auclair D, Taylor I, Gedrich R, Voznesensky A, Riedl B, Post LE, Bollag G, Trail PA 2004 BAY 439006 exhibits broad spectrum oral antitumor activity and targets the $R A F / M E K / E R K$ pathway and receptor tyrosine kinases involved in tumor progression and angiogenesis. Cancer Res 64:7099-7109.

17. Shimaoka K, Schoenfeld DA, DeWys WD, Creech RH, DeConti R 1985 A randomized trial of doxorubicin versus doxorubicin plus cisplatin in patients with advanced thyroid carcinoma. Cancer 56:2155-2160.

18. de Castroneves LA, Negrao MV, de Freitas RM, Papadia C, Lima JV Jr, Fukushima JT, Simao EF, Kulcsar MA, Tavares MR, Jorge AA, de Castro G Jr, Hoff PM, Hoff AO 2016 Sorafenib for the treatment of progressive metastatic medullary thyroid cancer: efficacy and safety analysis. Thyroid 26:414-419.

19. Lam ET, Ringel MD, Kloos RT, Prior TW, Knopp MV, Liang J, Sammet S, Hall NC, Wakely PE Jr, Vasko VV, Saji M, Snyder PJ, Wei L, Arbogast D, Collamore M, Wright JJ, Moley JF, Villalona-Calero MA, Shah MH 2010 Phase II clinical trial of sorafenib in metastatic medullary thyroid cancer. J Clin Oncol 28:2323-2330.

20. Ahmed M, Barbachano Y, Riddell A, Hickey J, Newbold KL, Viros A, Harrington KJ, Marais R, Nutting CM 2011 Analysis of the efficacy and toxicity of sorafenib in thyroid cancer: a Phase II study in a UK based population. Eur J Endocrinol 165:315-322.

21. Savvides $P$, Nagaiah G, Lavertu $P$, Fu P, Wright JJ, Chapman R, Wasman J, Dowlati A, Remick SC 2013 Phase II trial of sorafenib in patients with advanced anaplastic carcinoma of the thyroid. Thyroid 23:600-604.

22. Sugitani I, Miyauchi A, Sugino K, Okamoto T, Yoshida A, Suzuki S 2012 Prognostic factors and treatment outcomes for anaplastic thyroid carcinoma: ATC Research Consortium of Japan cohort study of 677 patients. World J Surg 36: 1247-1254.

Address correspondence to: Yasuhiro Ito, $M D, P h D$ Clinical Trial Management Center Kuma Hospital 8-2-35, Shimoyamate-dori Chuo-ku Kobe-shi Hyogo 650-0011 Japan

E-mail: ito01@kuma-h.or.jp 